\title{
Exploring a boundary-less cooperation approach for heterogeneous co-located networks
}

\author{
Eli De Poorter, Pieter Becue, Ingrid Moerman and Piet Demeester \\ Department of Information Technology (INTEC), Ghent University - IBBT \\ Gaston Crommenlaan 8 - bus 201, 9050 Gent, Belgium \\ Email: eli.depoorter@intec.ugent.be
}

\begin{abstract}
In a future 'internet of things', an increasing number of every-day objects are connected with each other. Nowadays, connectivity between these devices is supported by assigning each device to an existing (wireless) network. However, these networks do not take into account the individual needs of these devices, even though all these devices are very different in terms of application requirements and hardware capabilities. Moreover, multiple existing networks are often configured independent from each other without any interaction. As an alternative, this paper proposes and discusses a methodology that more efficiently supports network cooperation between heterogeneous devices. The paper argues for autonomously created communities of similar devices, that are able to negotiate with different co-located communities to further optimize their network performance. Different communities engage in cooperation by activating network services, but only when the end result is beneficial for all involved communities. In this paper, the concepts and advantages of this approach are discussed. In addition, a methodology is explored that is able to realize these concepts. Finally, based on this methodology, possible network solutions are presented, remaining challenges are listed and future research opportunities are identified.
\end{abstract}

Index Terms-Network negotiation, self-organization, network cooperation, network optimization, network coexistence.

\section{INTRODUCTION}

Several authors have predicted a future 'internet of things', in which an increasing number of objects are (wirelessly) connected with each other [1]. They predict that a large number of co-located every-day devices will be equipped with (wireless) communication interfaces. This trend can already be observed by looking at the rising popularity of wireless car ports, televisions, radios, rolling shutters and different types of household sensors. Allowing connectivity between objects results in increased interactivity with the environment, which in turn enables wireless next-generation applications [2], [3] such as wireless building automation, automated e-health solutions, interactive museum exhibitions and personalized entertainment systems.

However, current network solutions are not designed for the scale and complexity of these applications. Nowadays, devices are often manually configured and grouped together in different subnets based on their network technology. As a result, the same network configuration and policies are enforced to all the devices of a single subnet. However, future devices that use similar communication technologies can nevertheless have strongly diverging goals and capabilities.
As such, such an approach will no longer result in an optimal network performance for future heterogeneous networks [4].

Rather than using statically configured network boundaries, this paper explores a network cooperation methodology that enhances the performance of devices by forming spontaneous communities of like-minded devices. The central idea of the paper is that devices dynamically search for co-located devices with similar network preferences and (hardware and software) capabilities. This way, communities consisting of devices with similar network expectations are formed on an ad-hoc basis. Due to the similarities between the involved devices, it is easier to optimize the network performance of each community. In addition, different communities can cooperate with each other by making (software or hardware) resources available to other communities: after discovering co-located communities, the communities negotiate and select the optimal resources so that all involved communities benefit from cooperation.

The main goal of this explorative paper is to challenge the notion that network boundaries should be static. To this end, an alternative approach is presented that describes a new cross-network cooperation mechanism for heterogeneous colocated devices. The remainder of the paper is structured as follows. First, Section II defines the terms and concepts that will be used throughout the paper. Based on these concepts, Section III illustrates the advantages of boundary-less network cooperation in the form of an example. Section IV presents a methodology to realize these cooperation concepts. The related work from Section V gives an overview of related network approaches. Finally, Section VII concludes the paper.

\section{TERMINOLOGY}

In this section, the key components of the cooperation scheme are introduced.

\section{A. Symbiotic community}

A symbiotic community is defined as a set of nodes that have derived common incentives ('network goals') and which have an agreed-upon trust relation. Since all members of a community have similar network goals, the community can negotiate with other communities on behalf of all its member devices. Before joining a community, devices want to be sure they can trust all other community members. Devices that belong to the same owner are implicitly assumed to trust each other. Otherwise, a trust relationship can be established 
through the use of certificates which are issued by a trusted certification authority.

\section{B. Community Incentives}

Community incentives describe the expected benefits for a community when the community engages in symbiotic cooperation. Incentives do not express low-level performance metrics, but express the high-level network goals of each community. Communities will only engage in cooperation with other communities when the cooperation improves the performance of these network goals.

Example incentives are the following:

- HIGH_THROUGHPUT, HIGH_RELIABILITY or LOW_DELAY (to obtain better QoS guarantees)

- HIGH_NETWORK_LIFETIME (to prevent frequent battery replacement)

- HIGH_COVERAGE (to reach more clients)

- LOW_EXPOSURE (due to health regulations)

- GET_PUBLIC_ACCESS (to get internet connectivity)

\section{Symbiotic service}

A symbiotic service is a technique that realizes one or more of the community incentives. Thus, whereas incentives indicate network goals, symbiotic services are the means to realize these goals. A symbiotic service is not crucial for the correct working of the individual symbiotic communities, but can be activated or deactivated when cooperation with colocated communities is required. For example, a community can offer an internet access service to a second community, or an interference avoidance service [5] can be offered to optimize the performance of a neighboring community. Table I lists several example symbiotic services and their influence on the community incentives.

\section{Incentive driven networking}

Incentive driven networking is defined as the selection and activation of the optimal set of symbiotic services in each community with the goal to optimize the incentives of each participating community. Based on this definition, the distinction between symbiotic incentives and services can be thought about as follows: a symbiotic service can be activated or deactivated, whereas an incentive indicates a high-level application or management objective.

\section{ILLUSTRATION OF SYMBIOTIC NETWORKING}

Fig. 1. Without symbiotic networking different devices are either (i) connected to a single network (not shown on figure) or (ii) split into two networks that do not interact.

To illustrate how these concepts are used, consider the following example. A suspension bridge is monitored using battery-powered sensor devices [3] that use wireless IEEE 802.15.4 technology to communicate with each other. Two types of devices are installed:

- Seismic sensors monitor the force distribution and tension of different bridge components. When the measured
Fig. 2. With symbiotic networking, the incentives of the individual devices are taken into account.

values deviate strongly from the normal situation, a notification is forwarded immediately to a monitoring PC. To ensure a timely reaction in emergency situations, the devices have stringent delay and reliability incentives.

- A different type of (battery-powered) sensor devices monitors the number of vehicles that passes and measures the resulting pollution. Reports are sent periodically to the PC of the traffic department. To prevent frequent battery replacements, the main incentive of these battery-powered devices is the network lifetime.

When no symbiotic networking is supported, the sensor devices would either (i) be grouped together in one large network that does not take into account the incentives of the individual devices or (ii) be split into two optimized networks that do not interact (Figure 1).

However, when symbiotic networking is supported, a different approach is taken. Based on their incentives, the devices self-organize into two independent communities. The communities can chose to cooperate by selecting and activating the optimal network services in each community. By activating the interference avoidance service in community A, emergency packets from community B are transmitted more reliably [5]. When the packet sharing service in community $\mathrm{A}$ is also activated, packets from community $\mathrm{B}$ can be transmitted to any sink using more optimal routes, resulting in less delay. Similarly, by activating the aggregation service in community A, less packets need to be transmitted, thus improving the network lifetime of community A [6]. This way, symbiotic networking can improve the incentives of each individual device, even in small networks.

\section{SYMBIOTIC NETWORK COOPERATION}

To enable this type of boundary crossing optimizations, a methodological approach is explored in this section. The process consists of the following 5 phases, all of which will be discussed in more detail:

1) First, communities of similar devices are created.

2) The communities use varying communication technologies to discover each other.

3) After discovery, the communities negotiate about the optimal set of symbiotic services.

4) This is followed by the actual activation of the services.

5) Finally, the communities monitor if all services are actually deployed and if the communities behave correctly.

\section{A. Phase 1 - Community creation}

The first phase of the negotiation process partitions the devices into communities of directly connected devices with similar incentives. All devices that are part of a single community should be similar in terms of capabilities (such as available services) and incentives. In addition, they should trust each other (by for example using security certificates) and 


\begin{tabular}{|c|c|c|c|c|c|}
\hline \multirow[b]{2}{*}{ Symbiotic service } & \multirow[b]{2}{*}{ Description } & \multicolumn{4}{|c|}{ Expected influence on the incentives } \\
\hline & & Throughput & Delay & Reliability & $\begin{array}{l}\text { Network } \\
\text { Lifetime }\end{array}$ \\
\hline Shared routing & $\begin{array}{l}\text { Allows symbiotic communities to interpret and route packets from other } \\
\text { symbiotic communities }\end{array}$ & + & + & + & \pm \\
\hline Interference avoidance & $\begin{array}{l}\text { Symbiotic communities cooperate by selecting the transmission frequencies } \\
\text { which are least harmful for each other }\end{array}$ & + & \pm & + & \pm \\
\hline $\begin{array}{l}\text { Coordinated sleeping } \\
\text { schemes }\end{array}$ & $\begin{array}{l}\text { To conserve energy, the devices from the involved communities use matching } \\
\text { sleep schemes }\end{array}$ & - & - & \pm & + \\
\hline Packet aggregation & $\begin{array}{l}\text { To reduce the number of transmissions, multiple information exchanges are } \\
\text { aggregated into a single packet }\end{array}$ & + & - & - & + \\
\hline
\end{tabular}

TABLE I

EXAMPLE LIST OF SYMBIOTIC SERVICES AND THEIR INFLUENCE ON COMMUNITY INCENTIVES (+: POSITIVE INFLUENCE, -: NEGATIVE INFLUENCE, \pm : VARIABLE OR NO INFLUENCE)

they should be able to communicate with each other (either directly, or through intermediate devices that are part of the same community).

The partitioning of devices into separate symbiotic communities can occur either at-run-time or at design-time. As an example, the outline of a simple partitioning approach is presented in Algorithm 1. The algorithm assumes that the incentives, services and settings of each community are described using a standardized 'community profile', which is transmitted over a predetermined radio frequency. The algorithm starts when each individual device creates a community profile based on the requirements of its high-level applications. During the algorithm, devices search for co-located devices which are similar enough to be part of the same community. When no more compatbile co-located devices are found, the algorithm is finished. In environments that are strongly heterogeneous, a community might be as small as a single device.

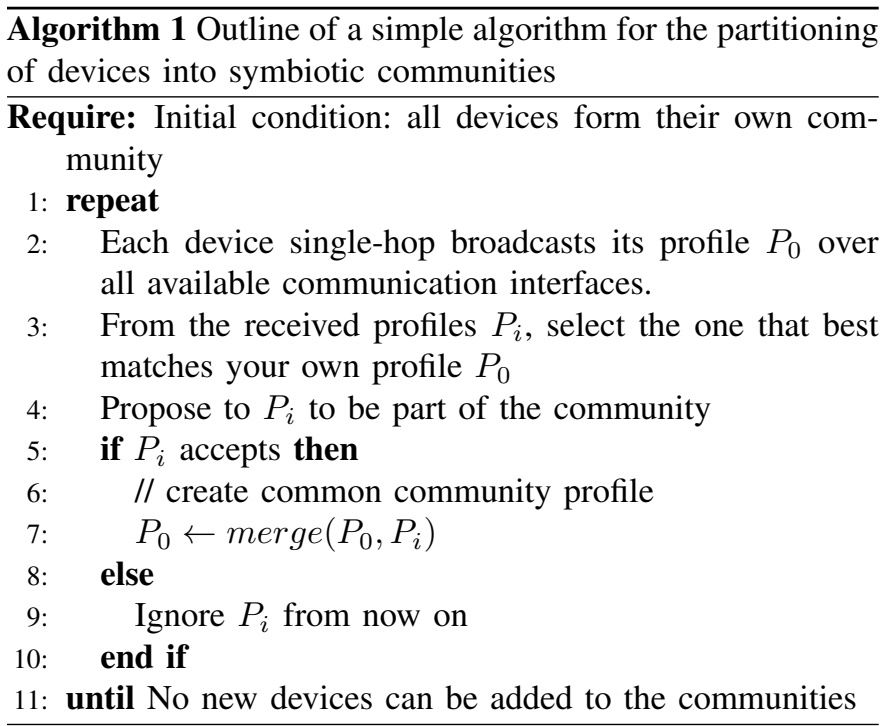

Whichever method is used, the end-result of this step is that all co-located devices are divided over different symbiotic communities consisting of devices with similar services and incentives. Since devices that form a community are very similar, their overall network performance can be strongly optimized based on the incentives of the community.

\section{B. Phase 2 - Community Discovery}

In the previous phase, devices were partitioned into separate communities with similar incentives and services. Now, the symbiotic communities find out if they are co-located with other communities capable of symbiotic networking. Community discovery consists of the following steps:

1) Assignation of discovery nodes. Each symbiotic community decides on the optimal number of devices that are needed to detect co-located communities. To bear minimal impact on the network performance, a subset of discovery nodes can suffice.

2) Community advertisement. Next, the communities broadcast beacon packets that identify their community ID. In addition, network settings are transmitted that describe how to contact the community. Using these settings, single hop communication between the discovery nodes of different communities is possible.

3) Profile exchange. The discovery nodes use the received information to exchange their community profile.

4) Forward the received profile. Finally, the received community profile is forwarded to the 'negotiation' entity of each community (see next section).

\section{Phase 3 - Community Negotiation}

By now, the symbiotic communities have exchanged profiles which describe the incentives of each community. The next steps investigate if cooperation between different co-located communities (in the form of activating cross-network services such as interference avoidance) is beneficial. To be able to participate in this step of symbiotic networking, each participating community should have a negotiation entity. This negotiation entity is either a single, central manager that is trusted by both communities or an entity that is distributed over several nodes of each community. Negotiation consists of the following phases:

1) Announcement of negotiation entity. The negotiation entity of each symbiotic community regularly announces its presence to all nodes of the community by broadcasting 'negotiation advertise' messages. 
2) Collection of Symbiotic Community Profiles. All received Symbiotic profiles are forwarded to the nearest negotiation entity where the negotiation process is initialized.

3) Determine an influence rating for each service. For each available symbiotic service, the negotiation manager determines how the activation of the available service will influence the incentives of each community. For example, enabling aggregation can increase the NETWORK_LIFETIME incentive by $30 \%$ [6]. To agree on estimated influence of network services, results can be used from (i) existing literature, from (ii) network simulators or from (iii) network monitoring agents.

4) Calculate optimal set of Symbiotic Services. Based upon these influence ratios, the negotiation entity calculates the optimal selection of services that should be activated.

Several negotiation approaches are possible, based on methods such as game theory, self-learning approaches or mathematical formulas. As an example, below a heuristic ILP formulation of the negotiation process is derived which can be applied to any number $N$ participating networks, using the notations from Table II.

Assuming:

$$
\sum_{i=0}^{I} I P_{i, a}=1, \quad \forall a=0 . . N
$$

Then maximize:

$$
\sum_{a=0}^{N} C P_{a} * \text { profit }_{a}
$$

subject to:

profit $_{a}=\sum_{i=0}^{I}\left[I P_{i, a} *\left\{1+\sum_{s=0}^{S} \sum_{b=0}^{N}\left(S A_{s, b} * S I_{i, a ; s, b}\right\}\right]\right.$

profit $_{a} \geq 1, \quad \forall a=0 . . N$

$S A_{s, b}= \begin{cases}1 & \text { if service } \mathrm{s} \text { is activated in network } \mathrm{b} \\ 0 & \text { if service } \mathrm{s} \text { is deactivated in network } \mathrm{b} .\end{cases}$

With:

$\begin{cases}N= & \text { the total number of communities participating in the } \\ & \text { negotiation process. } \\ I= & \text { the total number of incentives. } \\ S= & \text { the total number of available services. }\end{cases}$

Formula (1) enforces that the sum of the incentive priorities of each community is normalized to be equal to one. For example, consider the situation where two applications are running on node $a$. One application requires a maximal throughput, whereas the other requires a long network lifetime. Assuming both applications are equally important, the incentive priorities would be divided equally amongst both applications, resulting in $I P_{\text {throughput }, a}=I P_{\text {lifetime }, a}=0.5$.
Formula (2) specifies that the total profit function should be maximized. The total profit is calculated as the sum of the profits of all $\mathrm{N}$ communities weighted by their priority.

The profit of each individual community $a$ is calculated in Formula (3). The formula evaluates how each service influences the incentives of community $a$ when activated. Since initially no services are used $\left(S A_{s, b}=0, \forall s=0 . . S, \forall b=0 . . N\right)$ the profit without symbiotic networking equals one. When new services are added, these services increase or lower the value of the incentives (for example: using sleep schemes might improve the Network Lifetime incentive by $60 \%$ ). Depending on the priorities of the incentives, the resulting profit function can result in more or less profit for each community.

The condition described in Formula (4) ensures that the performance of none of the participating communities is degraded after cooperation. If no solution is found that results in better performance for a community $a$, this community will not participate in the cooperation. Optionally, condition (4) can be omitted when a community agrees to accept a decreased performance (for example to support nearby emergency networks).

The last condition in Formula (5) indicates that $S A_{s, b}$ are binary variables. After solving the ILP formulation, these indicate the services that maximally increase the objective function of all involved communities. In some cases, optimal symbiotic networking might require that the communities do not merge, but can result instead that both communities try to avoid each other through the use of interference avoidance mechanisms

Finally, based on the description of the Symbiotic services, constraints can be added. For example: if a network service needs to be activated over both communities (such as when using frequency hopping), the condition $S A_{\text {freqhop }, a}=$ $S A_{\text {freqhop }, b}$ is added.

\section{Phase 4 - Symbiotic Community Enabling}

After selecting the optimal set of service in each community, the next phase selects and activates the services in all involved communities. In addition, the settings of both networks are configured such that communication between the different networks is possible.

1) Propagation of the proposition. The negotiation entity forwards the decision about the service selection to the discovery nodes of the community. These devices know how to contact the other communities and relay the proposition to the co-located communities.

2) Additional negotiation (optional). Depending on the negotiation approach, the conclusions about the optimal set of services reached by both communities might differ. In this case, additional negotiation is required to find a set of services that both communities can agree on.

3) Confirmation and distribution. Once all involved communities agree on the service selection, the negotiation server distributes the chosen set of activated services and network settings in both communities. 


\begin{tabular}{ll}
\hline \hline Symbol & Meaning \\
\hline \hline$C P_{a}$ & $\begin{array}{l}\text { The priority of community a. Under normal operations, the priority of each community equals one. However, the performance of certified } \\
\text { emergency networks can be improved by giving them a higher priority, at the cost of a lower network performance of the other participating } \\
\text { communities. }\end{array}$ \\
profit ${ }_{a}$ & $\begin{array}{l}\text { The profit function of community a. This objective function should be maximized for each network to optimally profit from symbiotic } \\
\text { networking. }\end{array}$ \\
$I P_{i, a}$ & $\begin{array}{l}\text { The priority that is given to incentive i in community a. } \\
\text { The percentage by which incentive i from community a is improved when service } \mathrm{s} \text { is activated in community b. These values can be configured } \\
\text { at design-time, or monitoring agents can use learning techniques to intelligently monitor and change these percentages at run-time. }\end{array}$ \\
& $\begin{array}{l}\text { A binary variable ( } 0 \text { or } 1) \text { that indicates if service s is activated in community a. These variables are determined as the end result of the linear } \\
\text { program. }\end{array}$
\end{tabular}

TABLE II

LIST OF SYMBOLS.

4) Service migration (optional). If devices are missing certain symbiotic services, these services can be exchanged or installed remotely.

5) Activate the settings and services. Once both communities have received the optimal settings and services, the communities simultaneously switch to the selected configuration.

\section{E. Phase 5 - Policy enforcement}

Finally, communities will want to check if all other communities are 'playing by the rules', ie: are not cheating. For example, a monitoring agent can be used to (i) investigate if the selected services are actually activated and performing as expected and (ii) monitor the actual influence ratios of the activated services. If the set of services changes, or the measured influence ratios differ greatly from the influence ratios used in the negotiation, a new negotiation process is started. Figure 3 gives a general overview of the discussed symbiotic network methodology.

Fig. 3. The 6 phases of the symbiotic network methodology

\section{RELATED WORK}

Finally, this section gives an overview of related network cooperation approaches that are designed for a closer collaboration between different wireless networks and discusses the differences with the presented approach.

The use of a cognitive radio [7], [8] enables devices to autonomously reconfigure their transmission parameters based on the environment in which they operate. This allows the devices to reuse unused licensed spectrum without interfering with licensed users or to support an always best connected (ABC) paradigm. When parameters of the higher network layers are optimized based on changes in the network environment, the term cognitive networking [9] is used. A cognitive network is capable of perceiving current network conditions and use this information to plan, learn, and act according to end-to-end goals. Both cognitive approaches are focused on the optimization of a single protocol layer or a single device and do not usually involve negotiation or cooperation mechanics.
Whereas cognitive networking is designed for parameter optimization, in cooperative networks multiple devices work together towards reaching specific goals. For example, in [10] two MAC protocols are presented that use a relay node to store the packets that failed transmissions in previous time slots and attempts to retransmit them in an empty time slot. As a second example, when using opportunistic networking [11], the general idea is that, in the absence of a fixed infrastructure for connectivity, data exchanges could take place using the connection opportunities that arise due to impromptu encounters with other devices. Depending on the network layer that is optimized, different approaches are possible. However, barring some exceptions such as [12], most cooperation approaches have mainly considered one layer at a time.

Finally, service oriented architectures support automatic discovery of web services on newly discovered devices. Standards such as OWL-S [13] facilitate the automation of web service tasks including automated web service discovery, execution, interoperation, semantic descriptions, composition and execution monitoring. Unfortunately, these approaches are not designed to optimize networks at the lower network levels.

In conclusion, most of these cooperation approaches focus on improving the network at a single layer of the protocol stack. Also, connectivity between different independent networks is only allowed through the use of translation gateways at fixed locations. In contrast, our approach (i) takes into account the incentives of each individual device, (ii) is not limited to a single network layer, (iii) is designed to cope with heterogeneous devices and (iv) can cross network boundaries that are traditionally fixed. For optimal network coexistence, our approach can be combined with some of the mentioned techniques that are complementary to ours.

\section{FUTURE WORK}

The proposed methodology enables several interesting research opportunities:

- Solutions need to be developed to better estimate the influence of cross-network services on the network performance.

- New negotiation approaches can be developed based on game-theory or machine learning. 
- Network discovery can include methods for deducing and translating the network settings of neighboring communities, such as MAC type, packet types, used routing protocols and used communication settings.

- New addressing schemes can be designed that cope with dynamically created communities.

Future work will focus on the development of distributed and optimized algorithms for each of the described steps, as well as measuring the benefits of the outlined approach in a variety of network environments and technologies.

\section{CONCLUSION}

In conclusion, this paper presented a networking approach to support cooperation between heterogeneous networked devices. Rather than using manually configured (and timeconsuming) fixed network boundaries, network creation and negotiation is based on the concept of 'network incentives' or 'device goals'. The methodology comprises the following steps:

- Devices cluster together with other devices that have similar incentives, thus forming like-minded communities.

- Different communities broadcast their existence to each other.

- The communities exchange profiles which describe their available symbiotic services, their incentives and their network settings.

- A negotiation entity determines the optimal set of symbiotic services so that each participating community benefits from cooperation.

- Finally, the selected services are activated, so that the incentives of each community are improved.

An incentive driven networking paradigm has the potential to ultimately lead to a more efficient cross-layer optimization, resulting in better use of the scarce spectrum, better scalability, more efficient energy consumption, lower radio emissions and better QoS guarantees. The paper lists several interesting research opportunities for enterprising researchers and identified challenges, advantages and solutions for heterogeneous network cooperation.

\section{ACKNOWLEDGMENT}

This research is funded by the European Union's Seventh Framework Programme FP7/2007-2013 under grant agreement n 257542 (CONSERN project), by the IWT SBO SymbioNets project and the FWO G.0243.10 and FWO G.0291.09 grants.

\section{REFERENCES}

[1] "The internet of things," ITU Internet Reports, 2005.

[2] A. Wheeler, "Commercial applications of wireless sensor networks using zigbee," Comm. Mag., IEEE, vol. 45, no. 4, pp. 70 -77, apr. 2007.

[3] C. F. Garca-Hernndez, P. H. Ibargengoytia-Gonzlez, J. Garca-Hernndez, and J. A. Prez-Daz., "Wireless sensor networks and applications: a survey," IJCSNS International Journal of Computer Science and Network Security, VOL.7 No.3, March 2007.

[4] M. Yarvis, N. Kushalnagar, H. Singh, A. Rangarajan, Y. Liu, and S. Singh, "Exploiting heterogeneity in sensor networks," in Proceedings of the IEEE Infocom, 2005.
[5] L. Tytgat, B. Jooris, P. De Mil, E. De Poorter, I. Moerman, and P. Demeester, "Multichannel protocol for interference avoidance in wireless sensor networks," Proceedings of 2nd Gent University and KEIO University Global COE Joint workshop, 2008.

[6] E. De Poorter, S. Bouckaert, I. Moerman, and P. Demeester, "Nonintrusive aggregation in wireless sensor networks," Ad Hoc Networks Journal, vol. doi:10.1016/j.adhoc.2010.07.017, Article in Press, Available online 10 August 2010.

[7] S. Haykin, "Cognitive radio: brain-empowered wireless communications," Selected Areas in Communications, IEEE Journal on, vol. 23, no. 2, pp. $201-220$, feb. 2005.

[8] F. K. Jondral, "Software-defined radio - basics and evolution to cognitive radio," EURASIP Journal on Wireless Communications and Networking, vol. vol. 2005, no. 3, p. pp. 275283, 2005.

[9] R. W. Thomas, D. H. Friend, L. A. Dasilva, and A. B. Mackenzie, "Cognitive networks: adaptation and learning to achieve end-to-end performance objectives," Communications Magazine, IEEE, vol. 44, no. 12 , pp. $51-57$, dec. 2006.

[10] A. K. Sadek, K. Liu, and A. Ephremides, "Collaborative multiple-access protocols for wireless networks," ICC 2006.

[11] L. Pelusi, A. Passarella, and M. Conti, "Opportunistic networking: data forwarding in disconnected mobile ad hoc networks," Communications Magazine, IEEE, vol. vol.44, no.11, pp. pp.134-141, November 2006.

[12] J. Garca-Vidal, M. Guerrero-Zapata, J. Morillo, and D. Fust, "A protocol stack for cooperative wireless networks," Wireless Systems and Mobility in Next Generation Internet, In Lecture Notes in Computer Science (LNCS), vol. Volume 4396, pp. pp 62-73, 2007.

[13] "Owl-s: Semantic markup standard for web services, http://www.w3.org/submission/owl-s/." 